\title{
Magnetic-Field Density-Functional Theory
}

\section{(BDFT): Lessons from the Adiabatic Connection}

\author{
Sarah Reimann, ${ }^{*, \dagger}$ Alex Borgoo, ${ }^{*} \dagger$ Erik Tellgren, ${ }^{\dagger}$ Andrew M. Teale, ${ }^{\ddagger}$ and Trygve \\ Helgaker ${ }^{*} \dagger$ \\ $\dagger$ Department of Chemistry, Centre for Theoretical and Computational Chemistry, \\ University of Oslo, P.O. Box 1033, Blindern, Oslo N-0315, Norway \\ $\ddagger$ School of Chemistry, University of Nottingham, University Park, Nottingham NG7 2RD, \\ $U K$
}

E-mail: sarah.reimann@kjemi.uio.no; alex.borgoo@kjemi.uio.no; t.u.helgaker@kjemi.uio.no

\begin{abstract}
We study the effects of magnetic fields in the context of magnetic field densityfunctional theory (BDFT), where the energy is a functional of the electron density $\rho$ and the magnetic field $\mathbf{B}$. We show that this approach is a worthwhile alternative to current-density functional theory (CDFT) and may provide a viable route to the study of many magnetic phenomena using density-functional theory (DFT). The relationship between BDFT and CDFT is developed and clarified within the framework of the fourway correspondence of saddle functions and their convex and concave parents in convex analysis. By decomposing the energy into its Kohn-Sham components, we demonstrate that the magnetizability is mainly determined by those energy components that are related to the density. For existing density functional approximations, this implies that, for the magnetizability, improvements of the density will be more beneficial than introducing a magnetic-field dependence in the correlation functional. However, once a
\end{abstract}


good charge density is achieved, we show that high accuracy is likely only obtainable by including magnetic-field dependence. We demonstrate that adiabatic-connection (AC) curves at different field strengths resemble one another closely provided each curve is calculated at the equilibrium geometry of that field strength. By contrast, if all AC curves are calculated at the equilibrium geometry of the field-free system, then the curves change strongly with increasing field strength due to an increased static correlation of the compressed atoms. This holds also for density functional approximations, where we demonstrate that the main error encountered in the presence of a field is already present at zero field strength, indicating that density-functional approximations may be applied to systems in strong fields, without the need of treating additional static correlation.

\section{Introduction}

Magnetic fields and their effects on atoms and molecules have for many years been an active area of research in physics and chemistry. Of particular interest are molecular magnetic properties such as NMR shielding constants and magnetizabilities, which are measurable physical observables and an important application area of quantum chemistry. We also note an interest in the effects of ultrastrong magnetic fields on atoms and molecules in astrophysics. $^{1-3}$ From a theoretical point of view, the study of molecules in ultrastrong magnetic fields can give new insight, such as the recent discovery of a hitherto unknown perpendicular paramagnetic bonding mechanism. ${ }^{4}$

Regarding the computation of molecular magnetic properties, the performance of KohnSham density-functional theory (DFT) is still not satisfactory. ${ }^{5-7}$ For an improvement in density-functional approximations (DFAs), it is necessary to understand the effect of magnetic fields on the components of the Kohn-Sham energy - in particular, on the correlation functional. In this paper, we analyse for the first time the field dependence of the Kohn-Sham energy components, using the adiabatic connection (AC). 
There are two different ways of including the effects of magnetic fields in DFT. In currentdensity-functional theory (CDFT), a current dependence is introduced in the universal density functional, which then depends on the density and paramagnetic current density. ${ }^{8,9}$ In magnetic-field density-functional theory (BDFT), we introduce instead a field dependence in the density functional. ${ }^{10}$ Here, we develop CDFT and BDFT within a common frameworknamely, the four-way correspondence of conjugate saddle functions and their convex and concave parents, allowing us to relate and compare the CDFT and BDFT correlation functionals.

For the construction of exchange-correlation functionals, AC curves have in the past provided useful insight. ${ }^{11-18}$ However, so far, this has been done only in the absence of magnetic fields. To examine and understand the performance of exact DFT and of DFAs in magnetic fields, we extend the studies of $\mathrm{AC}$ curves to include magnetic fields. We consider two regimes: the weak-field regime to understand and model magnetic properties better and the strong-field regime to improve our understanding of fundamental physical and chemical concepts. We begin with weak fields, computing derivatives of the energy, relevant to molecular properties, using the finite-difference method. The energy and derivatives are decomposed into their Kohn-Sham components and analysed individually. We investigate to what extent a field dependence in the density functional may improve the computation of the magnetizability, which is proportional to the second derivative of the energy with respect to the magnetic field strength. Finally, we consider strong magnetic fields, studying AC curves for exact DFT and approximate DFAs under such conditions.

The remainder of the paper is organized as follows. The theory of Kohn-Sham DFT in magnetic fields is outlined in Section, comparing the BDFT and CDFT formalisms. In Section, we introduce the AC for BDFT, allowing for a detailed analysis of the correlation energy. Computational details are given in Section . Our results in the weak and strong field regimes are presented and discussed in Section . Section gives concluding remarks and directions for future work. 


\section{DFT in a magnetic field}

In this section, we discuss DFT in the presence of a magnetic field - in particular, we develop CDFT and BDFT within the framework of convex conjugation, setting up and relating the Hohenberg-Kohn and Lieb variation principles for these theories. We begin with a review of DFT.

\section{DFT in the absence of a magnetic field}

In the absence of a magnetic field, the electronic Hamiltonian of an $N$-electron system in an external scalar potential $v$ is given by

$$
H_{\lambda}(v)=T+\lambda W+\sum_{i=1}^{N} v\left(\mathbf{r}_{i}\right)
$$

where $T$ is the $N$-electron kinetic-energy operator and $W$ the $N$-electron two-electron repulsion operator. We have included in the Hamiltonian the interaction-strength parameter $\lambda \in[0,1]$, equal to one for the fully interacting (physical) system and zero for the noninteracting system. The ground-state energy, according to the Rayleigh-Ritz variation principle, is given by

$$
E_{\lambda}(v)=\inf _{\gamma \in \mathcal{D}_{N}} \operatorname{tr} \gamma H_{\lambda}(v)
$$

where $\mathcal{D}_{N}$ is the set of all normalized $N$-electron density matrices $\gamma$ of finite kinetic energy.

From the linearity of $H_{\lambda}(v)$ in $v$ in Eq. (1) and from the Rayleigh-Ritz variation principle in Eq. (2), it follows that $E_{\lambda}(v)$ is concave and upper semi-continuous in $v$. Concavity means that a linear interpolation never overestimates the energy, whereas upper semi-continuity means that the energy can jump up but not down as limits are taken. An upper semicontinuous concave function is known as closed concave. More strongly, the ground-state energy is continuous in $v$ (jumping neither up nor down as limits are taken) ${ }^{19}$ but this fact is not important for us here. 
From the closed concavity of the ground-state energy, it follows that there exists a conjugate function $F_{\lambda}(\rho)$, the universal density functional, such that ${ }^{19}$

$$
\begin{aligned}
& E_{\lambda}(v)=F_{\lambda}^{\bullet}(v) \stackrel{\text { def }}{=} \inf _{\rho \in X}\left[F_{\lambda}(\rho)+(v \mid \rho)\right], \\
& F_{\lambda}(\rho)=E_{\lambda}^{\circ}(\rho) \stackrel{\text { def }}{=} \sup _{v \in X^{*}}\left[E_{\lambda}(v)-(v \mid \rho)\right],
\end{aligned}
$$

where $(v \mid \rho)=\int v(\mathbf{r}) \rho(\mathbf{r}) \mathrm{d} \mathbf{r}$. Moreover, $F_{\lambda}$ is closed convex (lower semi-continuous and convex), meaning that $-F_{\lambda}$ is closed concave. Unlike the ground-state energy, however, $F_{\lambda}$ is not continuous but everywhere discontinuous. ${ }^{20}$ The set $X=L^{3}\left(\mathbb{R}^{3}\right) \cap L^{1}\left(\mathbb{R}^{3}\right)$ is the Banach space of densities (including all $v$ - and $N$-representable densities), while its dual $X^{*}=L^{3 / 2}\left(\mathbb{R}^{3}\right)+L^{\infty}\left(\mathbb{R}^{3}\right)$ contains the potentials (including all Coulomb potentials). ${ }^{19} \mathrm{We}$ refer to Eqs. (3) and (4) as the Hohenberg-Kohn and Lieb variation principles, respectively. We note that the DFT density functional of Eq. (4) may be written in the equivalent constrained-search form ${ }^{19,21}$

$$
F_{\lambda}(\rho)=\min _{\gamma \mapsto \rho} \operatorname{tr} \gamma(T+\lambda W)
$$

where the minimization is over all $\gamma \in \mathcal{D}_{N}$ with density $\rho$. A minimizing density always exists in Eq. (5).

In convex analysis, the convex conjugate of a function $f: X \rightarrow[-\infty, \infty]$ is the closed convex function $f^{*}: X^{*} \rightarrow[-\infty, \infty]$ defined by $f^{*}\left(x^{*}\right)=\sup _{x \in X}\left[f(x)+\left(x^{*} \mid x\right)\right]$. If $f$ is itself closed convex, then according to the biconjugation theorem $f(x)=f^{* *}(x)=$ $\sup _{x^{*} \in X^{*}}\left[f^{*}\left(x^{*}\right)+\left(x^{*} \mid x\right)\right]$; see Refs. 22 and 23. Adapting the formalism of convex conjugation to DFT, we have in Eqs. (3) and (4) introduced the concave skew conjugate $F_{\lambda}^{\bullet}$ of $F_{\lambda}$ and the convex skew conjugate $E_{\lambda}^{\circ}$ of $E_{\lambda}$, respectively. By the biconjugation theorem, these skew conjugate functions satisfy $E_{\lambda}=F_{\lambda}^{\bullet}=\left(E_{\lambda}^{\circ}\right)^{\bullet}=E_{\lambda}^{\circ \bullet}$ and $F_{\lambda}=E_{\lambda}^{\circ}=\left(F_{\lambda}^{\bullet}\right)^{\circ}=F_{\lambda}^{\bullet \circ}$. 


\section{Current-density-functional theory (CDFT)}

Consider now an electronic system in the presence of a magnetic field $\mathbf{B}$, represented by a vector potential $\mathbf{A}$ such that $\mathbf{B}=\nabla \times \mathbf{A}$. The electronic Hamiltonian now becomes

$$
H_{\lambda}(v, \mathbf{A})=T(\mathbf{A})+\lambda W+\sum_{i=1}^{N} v\left(\mathbf{r}_{i}\right)
$$

where the kinetic-energy operator takes the form

$$
T(\mathbf{A})=\frac{1}{2} \sum_{i=1}^{N}\left(-\mathrm{i} \boldsymbol{\nabla}_{i}+\mathbf{A}\left(\mathbf{r}_{i}\right)\right)^{2}
$$

This Hamiltonian is linear in $v$ but quadratic in $\mathbf{A}$ :

$$
H_{\lambda}(v, \mathbf{A})=H_{\lambda}(v)-\mathrm{i} \sum_{i=1}^{N} \mathbf{A}\left(\mathbf{r}_{i}\right) \cdot \nabla_{i}+\frac{1}{2} \sum_{i=1}^{N} A^{2}\left(\mathbf{r}_{i}\right)
$$

where $A=|\mathbf{A}|$. Since linearity is required to make the ground-state energy concave (and to set up DFT by conjugation), we change variables from $(v, \mathbf{A})$ to $(u, \mathbf{A})$ where $u=v+\frac{1}{2} A^{2}$ and introduce a Hamiltonian $\bar{H}_{\lambda}(u, \mathbf{A})$ that is linear in both potentials: ${ }^{24}$

$$
\bar{H}_{\lambda}(u, \mathbf{A})=H_{\lambda}(u)-\mathrm{i} \sum_{i=1}^{N} \mathbf{A}\left(\mathbf{r}_{i}\right) \cdot \nabla_{i}
$$

From the Rayleigh-Ritz variation principle and the linearity of the Hamiltonian, it follows that the resulting ground-state energy

$$
\mathcal{E}_{\lambda}(u, \mathbf{A})=\inf _{\gamma \in \mathcal{D}_{N}} \operatorname{tr} \gamma \bar{H}_{\lambda}(u, \mathbf{A})
$$

is closed concave. Invoking the biconjugation theorem and introducing the notation $\left(\mathbf{A} \mid \mathbf{j}_{\mathrm{p}}\right)=$ $\int \mathbf{A}(\mathbf{r}) \mathbf{j}_{\mathrm{p}}(\mathbf{r}) \mathrm{d} \mathbf{r}$ where $\mathbf{j}_{\mathrm{p}}$ is the paramagnetic current density, we arrive at the Hohenberg-Kohn 
and Lieb variation principles of current-density-functional theory (CDFT),

$$
\begin{aligned}
\mathcal{E}_{\lambda}(u, \mathbf{A}) & =\mathcal{F}_{\lambda}^{\bullet, \bullet}(u, \mathbf{A}) \\
& =\inf _{\rho, \mathbf{j}_{\mathrm{p}}}\left[\mathcal{F}_{\lambda}\left(\rho, \mathbf{j}_{\mathrm{p}}\right)+(u \mid \rho)+\left(\mathbf{A} \mid \mathbf{j}_{\mathrm{p}}\right)\right], \\
\mathcal{F}_{\lambda}\left(\rho, \mathbf{j}_{\mathrm{p}}\right) & =\mathcal{E}_{\lambda}^{\circ, \circ}\left(\rho, \mathbf{j}_{\mathrm{p}}\right) \\
& =\sup _{u, \mathbf{A}}\left[\mathcal{E}_{\lambda}(u, \mathbf{A})-(u \mid \rho)-\left(\mathbf{A} \mid \mathbf{j}_{\mathrm{p}}\right)\right] .
\end{aligned}
$$

The notation $\mathcal{F}_{\lambda}^{\bullet, \bullet}$ and $\mathcal{E}_{\lambda}^{\circ, \circ}$ is an obvious extension of the notation for convex and concave conjugates $F_{\lambda}^{\bullet}$ and $E_{\lambda}^{\circ}$ of univariate functions to bivariate functions, where conjugations with respect to the two variables are separated by a comma.

The closed convex CDFT density functional $\mathcal{F}_{\lambda}$ may be expressed in the equivalent constrained-search form

$$
\mathcal{F}_{\lambda}\left(\rho, \mathbf{j}_{\mathrm{p}}\right)=\inf _{\gamma \mapsto\left(\rho, \mathbf{j}_{\mathrm{p}}\right)} \operatorname{tr} \gamma(T+\lambda W)
$$

where we minimize over all $N$-electron density matrices $\gamma$ with density $\rho$ and paramagnetic current density $\mathbf{j}_{\mathrm{p}}$. CDFT was introduced by Vignale and Rasolt using the constrained-search formulation in $1987,{ }^{8,9}$ and formulated in terms of convex conjugation by Tellgren et al. in $2012 .{ }^{24}$

CDFT is considerably less developed than DFT, both formally and practically. KohnSham theory may be set up in the usual manner (see Ref. 24 for further discussion), but the $N$-representability problem is severe for single-orbital systems in CDFT and an open question

for two- and three-orbital systems. ${ }^{25}$ Unlike for DFT in Eq. (5), it is unknown whether a minimizing density matrix always exists in Eq. (13).

\section{Four-way correspondence of DFT in a magnetic field}

Instead of performing a full skew conjugation of $\mathcal{E}_{\lambda}$, with respect to both variables, as done in CDFT, we may perform partial skew conjugations, with respect to one of the two variables. 
The energy $\mathcal{E}_{\lambda}$ then becomes the partial skew conjugate to $\underline{\mathcal{G}}_{\lambda}$ and $\underline{\mathcal{H}}_{\lambda}$ in the manner

$$
\begin{array}{ll}
\underline{\mathcal{G}}_{\lambda}=\mathcal{E}_{\lambda}^{\circ}, & \mathcal{E}_{\lambda}=\underline{\mathcal{G}}_{\lambda}^{\bullet}, \\
\underline{\mathcal{H}}_{\lambda}=\mathcal{E}_{\lambda}^{\circ}, & \mathcal{E}_{\lambda}=\underline{\mathcal{H}}_{\lambda}^{\bullet},
\end{array}
$$

where $\underline{\mathcal{G}}_{\lambda}(\rho, \mathbf{A})$ is a convex-concave saddle function (convex in $\rho$ and concave in $\mathbf{A}$ ), whereas $\underline{\mathcal{H}}_{\lambda}\left(u, \mathbf{j}_{\mathrm{p}}\right)$ is a concave-convex saddle function (concave in $u$ and convex in $\mathbf{j}_{\mathrm{p}}$ ). Likewise, the CDFT universal density functional $\mathcal{F}_{\lambda}=\mathcal{E}_{\lambda}^{\circ, 0}$ is the partial skew conjugate to the convexconcave saddle function $\overline{\mathcal{G}}_{\lambda}(\rho, \mathbf{A})$ and the concave-convex saddle function $\overline{\mathcal{H}}_{\lambda}\left(u, \mathbf{j}_{\mathrm{p}}\right)$ in the manner

$$
\begin{aligned}
& \overline{\mathcal{G}}_{\lambda}=\mathcal{F}_{\lambda}^{\bullet} \quad \mathcal{F}_{\lambda}=\overline{\mathcal{G}}_{\lambda}^{, \circ}, \\
& \overline{\mathcal{H}}_{\lambda}=\mathcal{F}_{\lambda}^{\bullet}, \quad \mathcal{F}_{\lambda}=\overline{\mathcal{H}}_{\lambda}^{\circ} .
\end{aligned}
$$

As suggested by the notation, the conjugate saddle functions satisfy the inequalities ${ }^{23,26}$

$$
\underline{\mathcal{G}}_{\lambda} \leq \overline{\mathcal{G}}_{\lambda}, \quad \underline{\mathcal{H}}_{\lambda} \leq \overline{\mathcal{H}}_{\lambda} .
$$

Moreover, $\underline{\mathcal{G}}_{\lambda}$ and $\underline{\mathcal{H}}_{\lambda}$ are by construction lower semi-continuous in the convex variable, whereas $\overline{\mathcal{G}}_{\lambda}$ and $\overline{\mathcal{H}}_{\lambda}$ are upper semi-continuous in the concave variable. In general, conflicts for certain argument values may prevent a saddle function from being simultaneously lower semi-continuous in the convex variable and upper semi-continuous in the concave variable. We have not attempted here to establish whether or not $\underline{\mathcal{G}}_{\lambda}=\overline{\mathcal{G}}_{\lambda}$ and $\underline{\mathcal{H}}_{\lambda}=\overline{\mathcal{H}}_{\lambda}$ (which would imply that these functions are simultaneously lower semi-continuous in the convex variable and upper semi-continuous in the concave variable). In any case, this does not matter in the sense that $\underline{\mathcal{G}}_{\lambda}$ and $\overline{\mathcal{G}}_{\lambda}$ have the same skew conjugate and likewise for $\underline{\mathcal{H}}_{\lambda}$ and $\overline{\mathcal{H}}_{\lambda}$ (and therefore give the same results in the Hohenberg-Kohn and Lieb variation principles). Indeed, 
the sets

$$
\begin{aligned}
{\left[\mathcal{G}_{\lambda}\right] } & =\left\{\mathcal{G}_{\lambda} \text { convex-concave } \mid \underline{\mathcal{G}}_{\lambda} \leq \mathcal{G}_{\lambda} \leq \overline{\mathcal{G}}_{\lambda}\right\}, \\
{\left[\mathcal{H}_{\lambda}\right] } & =\left\{\mathcal{H}_{\lambda} \text { concave-convex } \mid \underline{\mathcal{H}}_{\lambda} \leq \mathcal{H}_{\lambda} \leq \overline{\mathcal{H}}_{\lambda}\right\}
\end{aligned}
$$

are equivalence classes of saddle functions with the same skew conjugates:

$$
\begin{aligned}
& \mathcal{E}_{\lambda}=\mathcal{G}_{\lambda}^{\bullet}, \quad \mathcal{F}_{\lambda}=\mathcal{G}_{\lambda}^{\circ}, \quad \forall \mathcal{G}_{\lambda} \in\left[\mathcal{G}_{\lambda}\right], \\
& \mathcal{E}_{\lambda}=\mathcal{H}_{\lambda}^{\circ \circ}, \quad \mathcal{F}_{\lambda}=\mathcal{H}_{\lambda}^{\bullet}, \quad \forall \mathcal{H}_{\lambda} \in\left[\mathcal{H}_{\lambda}\right] .
\end{aligned}
$$

Each $\mathcal{G}_{\lambda} \in\left[\mathcal{G}_{\lambda}\right]$ is said to be a closed saddle function and likewise for $\mathcal{H}_{\lambda} \in\left[\mathcal{H}_{\lambda}\right]$. In the following, we do not distinguish between the different elements of $\left[\mathcal{G}_{\lambda}\right]$, writing in all cases $\mathcal{G}_{\lambda}$ rather than $\underline{\mathcal{G}}_{\lambda}$ or $\overline{\mathcal{G}}_{\lambda}$.

The four-way correspondence between the closed saddle functions $\mathcal{G}_{\lambda}$ and $\mathcal{H}_{\lambda}$ and their closed convex and concave parents $\mathcal{F}_{\lambda}$ and $\mathcal{E}_{\lambda}$ is illustrated in Fig. 1 . Such a four-way correspondence may always be set up given a closed convex bivariate function $f$ or a closed concave bivariate function $e$; see the discussion by Rockafellar. ${ }^{26}$ In general, the convexconcave saddle functions $g$ and the concave-convex saddle functions $h$ whose parents are $f$ and $e$ belong to equivalence classes $[g]$ and $[h]$, which may or may not contain a single element.

\section{Magnetic-field density-functional theory (BDFT)}

As an alternative to CDFT, we describe in magnetic-field density-functional theory (BDFT) ${ }^{10}$

the atomic or molecular system in terms of the semi-universal density functional $\mathcal{G}_{\lambda}(\rho, \mathbf{A})$ rather than in terms of the universal $\operatorname{CDFT}$ density functional $\mathcal{F}_{\lambda}\left(\rho, \mathbf{j}_{\mathrm{p}}\right)$, using the partial 
conjugations in Eq. (14), yielding the following Hohenberg-Kohn and Lieb variation principles:

$$
\begin{aligned}
& \mathcal{E}_{\lambda}(u, \mathbf{A})=\mathcal{G}_{\lambda}^{\bullet}(u, \mathbf{A})=\inf _{\rho}\left[\mathcal{G}_{\lambda}(\rho, \mathbf{A})+(u \mid \rho)\right], \\
& \mathcal{G}_{\lambda}(\rho, \mathbf{A})=\mathcal{E}_{\lambda}^{\circ,}(\rho, \mathbf{A})=\sup _{u}\left[\mathcal{E}_{\lambda}(u, \mathbf{A})-(u \mid \rho)\right],
\end{aligned}
$$

with the equivalent constrained-search formulation

$$
\mathcal{G}_{\lambda}(\rho, \mathbf{A})=\min _{\gamma \mapsto \rho} \operatorname{tr} \gamma[T(A)+\lambda W]-\frac{1}{2}\left(A^{2} \mid \rho\right) .
$$

These variation principles are similar to the standard, field-free Hohenberg-Kohn and Lieb variation principles in Eqs. (3) and (4), respectively, the only difference being the explicit dependence of the energy and density functionals on the vector potential A. As such, BDFT is simpler than CDFT, which requires the optimization of $\left(\rho, \mathbf{j}_{\mathrm{p}}\right)$ in the Hohenberg-Kohn variation principle and of $(v, \mathbf{A})$ in the Lieb variation principle; see Eqs. (11) and (12).

According to Eq. (16), the BDFT and CDFT density functionals are related as

$$
\begin{aligned}
& \mathcal{G}_{\lambda}(\rho, \mathbf{A})=\mathcal{F}_{\lambda}^{, \bullet}(\rho, \mathbf{A})=\inf _{\mathbf{j}_{\mathrm{p}}}\left[\mathcal{F}_{\lambda}\left(\rho, \mathbf{j}_{\mathrm{p}}\right)+\left(\mathbf{A} \mid \mathbf{j}_{\mathrm{p}}\right)\right] \\
& \mathcal{F}_{\lambda}\left(\rho, \mathbf{j}_{\mathrm{p}}\right)=\mathcal{G}_{\lambda}^{, \circ}\left(\rho, \mathbf{j}_{\mathrm{p}}\right)=\sup _{\mathbf{A}}\left[\mathcal{G}_{\lambda}(\rho, \mathbf{A})-\left(\mathbf{A} \mid \mathbf{j}_{\mathrm{p}}\right)\right]
\end{aligned}
$$

It is an open question whether the semi-universal density functional of BDFT is easier or more difficult to model than the fully universal density functional of CDFT.

\section{Kohn-Sham decomposition in BDFT and CDFT}

Kohn-Sham theory conventionally employs a noninteracting system with integer occupation numbers corresponding to a Slater determinant. Less commonly, fractional occupation may be considered in ensemble formalisms. The Kohn-Sham decompositions of the CDFT and 
BDFT density functionals, respectively, are given by

$$
\begin{aligned}
& \mathcal{F}_{\lambda}\left(\rho, \mathbf{j}_{\mathrm{p}}\right)=\mathcal{T}_{\mathrm{s}}\left(\rho, \mathbf{j}_{\mathrm{p}}\right)+\lambda J(\rho)+\mathcal{F}_{\mathrm{xc}, \lambda}\left(\rho, \mathbf{j}_{\mathrm{p}}\right) \\
& \mathcal{G}_{\lambda}(\rho, \mathbf{A})=\mathcal{K}_{\mathrm{s}}(\rho, \mathbf{A})+\lambda J(\rho)+\mathcal{G}_{\mathrm{xc}, \lambda}(\rho, \mathbf{A}),
\end{aligned}
$$

where $\mathcal{T}_{\mathrm{s}}$ and $\mathcal{K}_{\mathrm{s}}$ are the noninteracting ensemble CDFT and BDFT density functionals. With fractional occupation allowed, $\mathcal{T}_{\mathrm{s}}=\mathcal{F}_{0}$ and $\mathcal{K}_{\mathrm{s}}=\mathcal{G}_{0}$. In the case of integer occupation, $\mathcal{T}_{\mathrm{s}} \geq \mathcal{F}_{0}$ and $\mathcal{K}_{\mathrm{s}} \geq \mathcal{G}_{0}$, with equality for typical, non-degenerate systems. The functional $J(\rho)$ in Eqs. (28) and (29) is the Hartree functional,

$$
J(\rho)=\iint \rho\left(\mathbf{r}_{1}\right) \rho\left(\mathbf{r}_{2}\right) r_{12}^{-1} \mathrm{~d} \mathbf{r}_{1} \mathrm{~d} \mathbf{r}_{2},
$$

whereas $\mathcal{F}_{\mathrm{xc}, \lambda}\left(\rho, \mathbf{j}_{\mathrm{p}}\right)$ and $\mathcal{G}_{\mathrm{xc}, \lambda}(\rho, \mathbf{A})$ are the CDFT and BDFT exchange-correlation energies, respectively. Expressing the ground-state energy $\mathcal{E}_{\lambda}(u, \mathbf{A})$ in terms of $\mathcal{F}_{\lambda}\left(\rho, \mathbf{j}_{\mathrm{p}}\right)$ and $\mathcal{G}_{\lambda}(\rho, \mathbf{A})$, we obtain

$$
\begin{aligned}
\mathcal{E}_{\lambda} & (u, \mathbf{A}) \\
& =\mathcal{T}_{\mathrm{s}}\left(\rho, \mathbf{j}_{\mathrm{p}}\right)+\lambda J(\rho)+\mathcal{F}_{\mathrm{xc}, \lambda}\left(\rho, \mathbf{j}_{\mathrm{p}}\right)+(u \mid \rho)+\left(\mathbf{j}_{\mathrm{p}} \mid \mathbf{A}\right), \\
& =\mathcal{K}_{\mathrm{s}}(\rho, \mathbf{A})+\lambda J(\rho)+\mathcal{G}_{\mathrm{xc}, \lambda}(\rho, \mathbf{A})+(u \mid \rho),
\end{aligned}
$$

where $\left(\rho, \mathbf{j}_{\mathrm{p}}\right)$ are the ground-state density and paramagnetic current density associated with $(u, \mathbf{A})$.

From Eqs. (13) and (25), we find that the noninteracting density functionals may be expressed in the constrained-search form

$$
\begin{aligned}
\mathcal{T}_{\mathrm{s}}\left(\rho, \mathbf{j}_{\mathrm{p}}\right) & =\inf _{\gamma \mapsto\left(\rho, \mathbf{j}_{\mathrm{p}}\right)} \operatorname{tr} \gamma T(\mathbf{A})-\left(\mathbf{A} \mid \mathbf{j}_{\mathrm{p}}\right)-\frac{1}{2}\left(A^{2} \mid \rho\right), \\
\mathcal{K}_{\mathrm{s}}(\rho, \mathbf{A}) & =\inf _{\gamma \mapsto \rho} \operatorname{tr} \gamma T(\mathbf{A})-\frac{1}{2}\left(A^{2} \mid \rho\right),
\end{aligned}
$$


which upon substitution in Eq. (31) shows that the sum of noninteracting kinetic energy and the exchange correlation energies in CDFT and BDFT are identical:

$$
\begin{aligned}
\inf _{\gamma \mapsto\left(\rho, \mathbf{j}_{\mathrm{p}}\right)} \operatorname{tr} \gamma T(\mathbf{A}) & +\mathcal{F}_{\mathrm{xc}}\left(\rho, \mathbf{j}_{\mathrm{p}}\right) \\
& =\inf _{\gamma \mapsto \rho} \operatorname{tr} \gamma T(\mathbf{A})+\mathcal{G}_{\mathrm{xc}}(\rho, \mathbf{A}) .
\end{aligned}
$$

Whereas the minimizing noninteracting density matrix in CDFT reproduces both the density $\rho$ and the paramagnetic current density $\mathbf{j}_{\mathbf{p}}$ of the physical system, the corresponding density matrix in BDFT is required only to reproduce the density $\rho$. As a result, the noninteracting mechanical kinetic energy in CDFT is an upper bound to the corresponding kinetic energy in BDFT:

$$
\inf _{\gamma \mapsto\left(\rho, \mathbf{j}_{\mathrm{p}}\right)} \operatorname{tr} \gamma T(\mathbf{A}) \geq \inf _{\gamma \mapsto \rho} \operatorname{tr} \gamma T(\mathbf{A})
$$

From Eq. (34), it then follows that the BDFT exchange-correlation energy is an upper bound to the corresponding CDFT exchange--correlation energy:

$$
\mathcal{G}_{\mathrm{xc}, \lambda}(\rho, \mathbf{A}) \geq \mathcal{F}_{\mathrm{xc}, \lambda}\left(\rho, \mathbf{j}_{\mathrm{p}}\right)
$$

where $\left(\rho, \mathbf{j}_{\mathrm{p}}\right)$ are the ground-state density and paramagnetic current density associated with $(u, \mathbf{A})$.

Since in BDFT the noninteracting density matrix $\gamma$ of Eq. (33) needs only reproduce the density $\rho$ (as in standard DFT), Kohn-Sham BDFT does not suffer from the $N$-representability problem of Kohn-Sham CDFT, where the noninteracting density matrix $\gamma$ of Eq. (32) must also reproduce the paramagnetic current density $\mathbf{j}_{\mathrm{p}}$. 


\section{Adiabatic connection}

To study of the AC in BDFT, we generalizing the procedure of Teale, Coriani and Helgaker ${ }^{18}$ to non-zero field strengths. In this manner, high-accuracy AC curves may be generated using accurate wave functions, as will be discussed in Section . The resulting AC curves give valuable information about the role of dynamical and static correlation in BDFT. They may also be used to benchmark approximate DFAs, whose AC curves are obtained by scaling, as discussed in Section

\section{Adiabatic connection for BDFT}

We have formulated BDFT in terms of the gauge-dependent vector potential A. To connect with the original BDFT formulation by Grayce and Harris, ${ }^{10}$ we may rely on gauge invariance to write

$$
\begin{aligned}
& E_{\lambda}(v, \mathbf{B})=\mathcal{E}_{\lambda}\left(v-\frac{1}{2} A^{2}, \mathbf{A}\right), \\
& G_{\lambda}(\rho, \mathbf{B})=\mathcal{G}_{\lambda}(\rho, \mathbf{A})+\frac{1}{2}\left(\rho \mid A^{2}\right), \\
& K_{\mathbf{S}}(\rho, \mathbf{B})=\mathcal{K}_{\mathbf{s}}(\rho, \mathbf{A})+\frac{1}{2}\left(\rho \mid A^{2}\right),
\end{aligned}
$$

where $\mathbf{B}=\nabla \times \mathbf{A}$ is the magnetic field. Note that $\mathcal{E}_{\lambda}$ is a functional of $u$, while $E_{\lambda}$ is a functional of $v$. These definitions yield the following BDFT Hohenberg-Kohn and Lieb variation principles, respectively:

$$
\begin{aligned}
& E_{\lambda}(v, \mathbf{B})=\inf _{\rho}\left[G_{\lambda}(\rho, \mathbf{B})+(v \mid \rho)\right], \\
& G_{\lambda}(\rho, \mathbf{B})=\sup _{v}\left[E_{\lambda}(v, \mathbf{B})-(v \mid \rho)\right] .
\end{aligned}
$$

In this formulation of BDFT, all quantities are manifestly gauge invariant.

To set up the AC of BDFT, we proceed in the usual manner, rewriting the density 
functional as

$$
G_{1}(\rho, \mathbf{B})=K_{\mathrm{s}}(\rho, \mathbf{B})+\int_{0}^{1} \frac{\mathrm{d} G_{\lambda}(\rho, \mathbf{B})}{\mathrm{d} \lambda} \mathrm{d} \lambda .
$$

This expression is rigorous in the ensemble case, when $K_{\mathrm{s}}(\rho, \mathbf{B})=G_{0}(\rho, \mathbf{B})$, and relies on an approximation or additional non-degeneracy assumption in the case of integer occupation. Assuming that a maximizing potential $v$ exists in the Lieb variation principle of Eq. (39), we obtain

$$
G_{\lambda}(\rho, \mathbf{B})=\operatorname{tr} \gamma_{\lambda}^{\rho, \mathbf{B}} H_{\mu}(0, \mathbf{B})
$$

where $\gamma_{\lambda}^{\rho, \mathbf{B}}$ is the ground-state density matrix for the maximizing potential. By the HellmannFeynman theorem, we then obtain the following expression for the BDFT density functional:

$$
G_{1}(\rho, \mathbf{B})=K_{\mathrm{s}}(\rho, \mathbf{B})+\int_{0}^{1} \operatorname{tr} \gamma_{\lambda}^{\rho, \mathbf{B}} W \mathrm{~d} \lambda .
$$

Introducing the BDFT exchange and correlation functionals by

$$
\begin{aligned}
& E_{\mathrm{x}}(\rho, \mathbf{B})=\operatorname{tr} \gamma_{0}^{\rho, \mathbf{B}} W-J(\rho), \\
& E_{\mathrm{c}}(\rho, \mathbf{B})=\int_{0}^{1} \mathcal{W}_{\lambda}(\rho, \mathbf{B}) \mathrm{d} \lambda
\end{aligned}
$$

in terms of the field-dependent AC correlation integrand

$$
\mathcal{W}_{\mathrm{c}, \lambda}(\rho, \mathbf{B})=\operatorname{tr}\left(\gamma_{\lambda}^{\rho, \mathbf{B}}-\gamma_{0}^{\rho, \mathbf{B}}\right) W,
$$

we arrive at the BDFT density functional

$$
G_{1}(\rho, \mathbf{B})=T_{\mathrm{s}}(\rho, \mathbf{B})+J(\rho)+E_{\mathrm{xc}}(\rho, \mathbf{B}),
$$


where $E_{\mathrm{xc}}(\rho, \mathbf{B})=E_{\mathrm{x}}(\rho, \mathbf{B})+E_{\mathrm{c}}(\rho, \mathbf{B})$.

We here study the BDFT correlation energy in Eq. (46) by calculating the monotonically decreasing BDFT correlation integrand $\mathcal{W}_{\mathrm{c}, \lambda}(\rho, \mathbf{B})$ for interaction strengths in the interval $[0,1]$ by means of the Lieb variation principle. The quality of the AC integrand is determined

by the quality of the underlying wave-function model used for $E_{\lambda}(v, \mathbf{B})$ in the Lieb variation principle in Eq. (43.)

\section{AC curves for approximate density functionals}

Given the wealth of existing approximations for $E_{\mathrm{c}}(\rho)$ in DFT, we may consider developing approximations that generalize existing forms to $G_{\mathrm{c}}(\rho, \mathbf{B})$ in BDFT (if only by neglecting its field dependence). To compare and evaluate such approximate functionals against the benchmark AC data, uniform scaling relations may be employed.

For any explicit approximate functional, AC curves can be computed using the general formula $^{27}$

$$
\mathcal{W}_{\mathrm{c}, \lambda}\left(\rho_{1 / \lambda}, \mathbf{B}\right)=\frac{\partial}{\partial \lambda}\left(\lambda^{2} E_{\mathrm{c}}\left(\rho_{1 / \lambda}, \mathbf{B}\right)\right)
$$

where

$$
\rho_{1 / \lambda}(\mathbf{r})=\lambda^{-3} \rho(\mathbf{r} / \lambda)=\lambda^{-3} \rho\left(\mathbf{r}^{\prime}\right)
$$

in terms of the scaled coordinate by $\mathbf{r}^{\prime}=\mathbf{r} / \lambda$. For a field-dependent correlation functional that depends locally on the density, the correlation energy can be obtained as

$$
\begin{aligned}
E_{\mathrm{c}}\left(\rho_{1 / \lambda}, \mathbf{B}\right) & =\int \epsilon_{\mathrm{c}}\left(\rho_{1 / \lambda}(\mathbf{r}), \mathbf{B}\right) \mathrm{d} \mathbf{r} \\
& =\lambda^{3} \int \epsilon_{\mathrm{c}}\left(\lambda^{-3} \rho\left(\mathbf{r}^{\prime}\right), \mathbf{B}\right) \mathrm{d} \mathbf{r} .
\end{aligned}
$$

For functionals that also depend locally on the gradient, we have that

$$
\frac{\partial \rho_{1 / \lambda}(\mathbf{r})}{\partial \mathbf{r}}=\lambda^{-3} \frac{\partial \rho\left(\mathbf{r}^{\prime}\right)}{\partial \mathbf{r}}=\lambda^{-3} \frac{\partial \rho\left(\mathbf{r}^{\prime}\right)}{\partial \mathbf{r}^{\prime}} \frac{\partial \mathbf{r}^{\prime}}{\partial \mathbf{r}}=\lambda^{-4} \frac{\partial \rho\left(\mathbf{r}^{\prime}\right)}{\partial \mathbf{r}^{\prime}}
$$


At the LDA and GGA levels of theory, standard functionals may be employed, neglecting the field dependence.

For meta-GGA functionals, the kinetic-energy density is also needed. From Eq. (50), it follows that the orbitals and their derivatives scale as

$$
\begin{aligned}
\phi_{i}^{1 / \lambda}(\mathbf{r}) & =\lambda^{-3 / 2} \phi_{i}\left(\mathbf{r}^{\prime}\right) \\
\frac{\partial \phi_{i}^{1 / \lambda}(\mathbf{r})}{\partial \mathbf{r}} & =\lambda^{-\frac{3}{2}} \frac{\partial \mathbf{r}^{\prime}}{\partial \mathbf{r}} \frac{\partial \phi\left(\mathbf{r}^{\prime}\right)}{\partial \mathbf{r}^{\prime}}=\lambda^{-\frac{5}{2}} \frac{\partial \phi\left(\mathbf{r}^{\prime}\right)}{\partial \mathbf{r}^{\prime}}
\end{aligned}
$$

implying that the kinetic-energy density $\tau_{0}$ transforms as

$$
\tau_{0}^{1 / \lambda}(\mathbf{r})=\frac{1}{2} \sum_{i=0}^{\text {occ }}\left|\frac{\partial \phi_{i}^{1 / \lambda}(\mathbf{r})}{\partial \mathbf{r}}\right|^{2}=\lambda^{-5} \tau_{0}\left(\mathbf{r}^{\prime}\right)
$$

In a magnetic field, the kinetic-energy density must be modified to ensure gauge invariance. One such modification is to use the physical kinetic-energy density proposed by Maximoff and Scuseria, ${ }^{28}$ here given in the scaled form

$$
\tau_{\text {phys }}^{1 / \lambda}(\mathbf{r})=\frac{1}{2} \sum_{l}\left|(-i \nabla+\mathbf{A}(\mathbf{r})) \phi_{l}^{1 / \lambda}(\mathbf{r})\right|^{2}
$$

Finally, from $\mathbf{A}^{1 / \lambda}(\mathbf{r})=\lambda^{-1} \mathbf{A}\left(\mathbf{r}^{\prime}\right)$, we obtain $\tau_{\text {phys }}^{1 / \lambda}(\mathbf{r})=\lambda^{-5} \tau_{\text {phys }}\left(\mathbf{r}^{\prime}\right)$. The ACs for meta-GGAs can then be obtained by using the scaled density in Eq. (51) along with the scaled gradient in Eq. (52) and the scaled kinetic-energy density in Eq. (55), replacing $\tau_{0}$ by $\tau_{\text {phys }}$.

\section{Computational details}

Except as noted, all calculations have been performed using the LONDON quantum-chemistry software. ${ }^{29,30}$ For evaluation of the exchange-correlation functionals, this code uses the XCFun library. ${ }^{31}$ To ensure gauge-origin independence, London atomic orbitals ${ }^{32}$ are used throughout. Unless otherwise stated, we use the aug-cc-pVTZ basis set of Dunning and coworkers ${ }^{33,34}$ in 
Cartesian rather than spherical-harmonic form. This basis is used for both the orbital and potential expansions in the Lieb optimizations, for which the second-order scheme of Refs. 35,36 is employed, with a singular-value decomposition of $10^{-6}$ a.u. for the Hessian.

We have used bond lengths $1.4 \mathrm{bohr}$ for $\mathrm{H}_{2}, 5.7 \mathrm{bohr}$ for $\mathrm{He}_{2}$, and $3.028 \mathrm{bohr}$ for $\mathrm{LiH}$. The geometries for $\mathrm{HF}, \mathrm{H}_{2} \mathrm{O}, \mathrm{NH}_{3}, \mathrm{CH}_{4}, \mathrm{CO}$, and $\mathrm{F}_{2}$ are from Refs. 5,6, optimized at the $\operatorname{CCSD}(\mathrm{T}) / \mathrm{cc}-\mathrm{pVTZ}$ level of theory.

\section{Results and discussion}

This section consists of two parts. First, in Sec., we explore the weak-field regime by studying the magnetizability for a number of atomic and molecular systems. In Sec., we study the AC at different field strengths for $\mathrm{H}_{2}$ and $\mathrm{LiH}$, yielding insight into the magnetic-field dependence of the correlation energy up to a field strength of one atomic unit, $B_{0}=2.35 \times 10^{5} \mathrm{~T}$. In both subsections, the performance of various DFAs is assessed by comparing with accurate Kohn-Sham values, obtained at the full-configuration-interaction (FCI) level of theory using the Lieb variation principle.

\section{Magnetizability}

For the singlet closed-shell atomic and molecular systems considered here, the magnetizability,

$$
\xi=-\left.\frac{\mathrm{d}^{2} E(B)}{\mathrm{d} B^{2}}\right|_{B=0},
$$

describes the behaviour of the system in the weak-field regime: $E(B)=-\frac{1}{2} \xi B^{2}+\cdots$. Before considering $\xi$ itself, we consider in Sec. the dependence of the total electronic energy and its Kohn-Sham components on the magnetic field. Next, we calculate $\xi$ and its Kohn-Sham components accurately for some small systems in Sec., comparing with standard DFAs in Sec. . We conclude by considering the importance of the field dependence of the BDFT correlation functional in Sec. . 


\section{Energy of $\mathrm{H}_{2}$ in a perpendicular magnetic field}

In Fig. 2, we have plotted the FCI/aug-cc-pVTZ energy of $\mathrm{H}_{2}$ and its Kohn-Sham components against the strength of a perpendicular magnetic field, relative to the corresponding zero-field values. The plot covers a wide field range, up to $0.03 B_{0}$ (about $7000 \mathrm{~T}$ ). The calculated energies correspond to the points shown in the figure. For the total energy, the curves joining these points are plots of $E(B)-E(0) \approx-\frac{1}{2} \xi B^{2}-E(0)$, the curvature of which is $-\xi$. Similar functions are plotted for the Kohn-Sham energy components as a function of field. In Table 1, we have listed the total energy and the Kohn-Sham components at zero field and at the highest plotted field strength of $0.03 B_{0}$. All calculations have been carried out at the zero-field equilibrium geometry of $\mathrm{H}_{2}$.

The total energy of $\mathrm{H}_{2}$ increases diamagnetically in the field, with a large positive contribution from $T_{\mathbf{S}}(\rho, \mathbf{B})$, a large negative contribution from $(v \mid \rho)$, and smaller but still large contributions from $J(\rho)>0$ and $E_{\mathrm{x}}(\rho, \mathbf{B})<0$. The Kohn-Sham correlation energy $E_{\mathrm{c}}(\rho, \mathbf{B})$ makes a much smaller (negative) contribution. For this particular system, the Hartree energy $J(\rho)$ changes in a manner that closely follows that of the total energy.

While the increase in $T_{\mathrm{s}}(\rho, \mathbf{B})$ in the field is a reflection of the induced precessional motion of the electrons (closed-shell diamagnetism), the increase in $J(\rho)$ and decrease in $(v \mid \rho)$ and $E_{\mathrm{x}}(\rho, \mathbf{B})$ follows from the concomitant contraction of the density in the field.

We note that the second-order curve $E(B) \approx-\frac{1}{2} \xi B^{2}$ describes the variation of the energy (and likewise for the Kohn-Sham components) with $B$ to a remarkably high accuracy over the full field range in Fig. 2.

Table 1: The total electronic energy and its Kohn-Sham components of $\mathrm{H}_{2}$ at zero field and at $B=0.03 B_{0}$ (perpendicular) calculated at the FCI/aug-cc-pVTZ level (atomic units).

\begin{tabular}{c|cccccc}
\hline \hline & $E$ & $T_{\mathrm{s}}$ & $J$ & $(v \mid \rho)$ & $E_{\mathrm{x}}$ & $E_{\mathrm{c}}$ \\
\hline$B=0.00$ & -1.8870 & 1.1381 & 1.3217 & -3.6461 & -0.6438 & -0.0569 \\
$B=0.03$ & -1.8867 & 1.1389 & 1.3220 & -3.6468 & -0.6439 & -0.0569 \\
\hline \hline
\end{tabular}




\section{FCI Kohn-Sham magnetizabilities}

Table 2 contains the magnetizability $\xi$ and its Kohn-Sham components for $\mathrm{H}_{2}$, $\mathrm{He}, \mathrm{He}_{2}$, and Be calculated at the FCI/aug-cc-pVTZ level of theory and for LiH at the FCI/cc-pVTZ level for theory. For the diatomic systems, the field direction is perpendicular to the molecular axis. All derivatives have been obtained by finite difference, using the LONDON code for different values of the magnetic field.

Table 2: The magnetizability $\xi$ and its Kohn-Sham components calculated using FCI/aug-ccpVTZ theory (FCI/cc-pVTZ for LiH) (atomic units and perpendicular field)

\begin{tabular}{l|rrrrrr}
\hline \hline & $\xi$ & $\xi_{T_{\mathrm{s}}}$ & \multicolumn{1}{c}{$\xi_{J}$} & \multicolumn{1}{c}{$\xi_{v}$} & $\xi_{\mathrm{x}}$ & \multicolumn{1}{c}{$\xi_{\mathrm{c}}$} \\
\hline $\mathrm{H}_{2}$ & -0.763 & -1.877 & -0.751 & 1.475 & 0.375 & 0.014 \\
$\mathrm{He}$ & -0.399 & -1.197 & -0.434 & 1.019 & 0.217 & -0.004 \\
$\mathrm{He}_{2}$ & -0.798 & -2.392 & -0.874 & 2.043 & 0.434 & -0.008 \\
$\mathrm{Be}$ & -2.721 & -8.032 & -6.380 & 10.324 & 1.221 & 0.162 \\
$\mathrm{LiH}$ & -2.010 & -5.254 & -5.467 & 6.851 & 1.915 & -0.063 \\
\hline \hline
\end{tabular}

The magnitudes and signs of the Kohn-Sham contributions to the magnetizability $\xi$ in Table 2 are as expected from the discussion in Sec. . In particular, electron correlation makes the smallest contribution to $\xi$, with $\xi_{\mathrm{c}}$ ranging from $-1 \%$ for He to $6 \%$ for Be.

It is worth commenting on the magnetizability of the ${ }^{1} \mathrm{~S}$ atoms He and Be. For these atoms, there is no paramagnetic contribution to $\xi$, which is a simple expectation value $\xi_{T_{\mathrm{s}}}^{\text {dia }}=-\frac{1}{6} \int \rho(\mathbf{r}) r^{2} \mathrm{~d} \mathbf{r}$. In our finite-difference calculations, this term enters as part of the kinetic-energy contribution $\xi_{T_{\mathrm{s}}}$, which also contains non-vanishing canonical kinetic-energy contribution $\xi_{T_{\mathrm{s}}}^{\mathrm{can}}$. The sum of the contributions to the magnetizability from the canonical kinetic energy and all other Kohn-Sham terms vanishes for ${ }^{1} \mathrm{~S}$ atoms: $\xi_{T_{\mathrm{s}}}^{\mathrm{can}}+\xi_{J}+\xi_{v}+\xi_{\mathrm{x}}+\xi_{\mathrm{c}}=0$.

\section{DFA Kohn-Sham magnetizabilities}

Table 3 contains, for the PBE, BLYP, B97, and TPSS exchange-correlation functionals, the errors in the Kohn-Sham contributions to the magnetizability of $\mathrm{H}_{2}$ relative to the FCI/aug-cc-pVTZ contributions in Table 2. 
The use of an approximate exchange-correlation functional affects the magnetizability of an atom or a molecule in two ways. First, it gives an error in the optimized ground-state density in the Hohenberg-Kohn variation principle; second, it gives an error in the exchangecorrelation contribution to the magnetizability calculated from a given density. With this in mind, we have in Table 2 listed the errors in the Kohn-Sham components (relative to the FCI values) calculated both from the FCI ground-state density and from the ground-state density optimized using the given approximate exchange-correlation functional.

Table 3: The magnetizability $\xi$ and its Kohn-Sham components for $\mathrm{H}_{2}$ in a perpendicular magnetic field, calculated using FCI theory and using various DFAs with the FCI density and the DFA density (atomic units). All DFA values are relative to the FCI values. For TPSS, we work with $\tau_{\text {phys }}$ of Eq. (56).

\begin{tabular}{ll|ccccc}
\hline \hline & & $\xi$ & $\xi_{T_{\mathrm{s}}}$ & $\xi_{J}$ & $\xi_{v}$ & $\xi_{\mathrm{xc}}$ \\
\hline FCI & & -0.763 & -1.877 & -0.751 & 1.475 & 0.390 \\
\hline$\Delta \mathrm{PBE}$ & $@ \rho_{\mathrm{FCI}}$ & -0.036 & 0 & 0 & 0 & -0.037 \\
$\Delta \mathrm{BLYP}$ & $@ \rho_{\mathrm{FCI}}$ & -0.033 & 0 & 0 & 0 & -0.034 \\
$\Delta \mathrm{B} 97$ & $@ \rho_{\mathrm{FCI}}$ & -0.024 & 0 & 0 & 0 & -0.025 \\
$\Delta \mathrm{TPSS}$ & $@ \rho_{\mathrm{FCI}}$ & -0.016 & 0 & 0 & 0 & -0.017 \\
\hline$\Delta \mathrm{PBE}$ & $@ \rho_{\mathrm{PBE}}$ & -0.022 & -0.065 & -0.069 & +0.102 & +0.010 \\
$\Delta \mathrm{BLYP}$ & $@ \rho_{\mathrm{BLYP}}$ & -0.025 & -0.071 & -0.073 & +0.110 & +0.009 \\
$\Delta \mathrm{B} 97$ & $@ \rho_{\mathrm{B} 97}$ & -0.011 & -0.030 & -0.039 & +0.051 & +0.007 \\
$\Delta \mathrm{TPSS}$ & $@ \rho_{\mathrm{TPSS}}$ & -0.002 & -0.015 & -0.027 & +0.030 & +0.011 \\
\hline \hline
\end{tabular}

From Table 2, we see that the use of the FCI ground-state density gives an error in $\xi$ ranging from -0.016 a.u. (2\%) for the TPSS functional to -0.036 a.u. (5\%) for the PBE functional. Since the FCI density is used, the errors arise only from the exchange-correlation functional, all other Kohn-Sham terms having been treated exactly. The error of the approximate exchange-correlation functionals ranges from $5 \%$ to $10 \%$.

Perhaps surprisingly, for each approximate exchange-correlation functional in Table 2, the error in $\xi$ reduces when calculated from the ground-state density optimized with the same functional, ranging from only -0.002 a.u. for the TPSS functional to -0.025 a.u. for the BLYP functional. Although the error in $\xi_{T_{\mathrm{s}}}+\xi_{J}+\xi_{v}$ increases in magnitude in all cases (by -0.012 a.u. for TPSS and -0.034 a.u. for BLYP), this increase is more than compensated 
for by a decrease in the error in $\xi_{\mathrm{xc}}$ (by +0.028 a.u. for TPSS and +0.043 a.u. for BLYP). Clearly, to improve on the calculated magnetizabilities in DFT, the exchange-correlation functionals must both give better zero-field ground-state densities and describe the changes in the exchange-correlation energy that occur when a magnetic field is applied.

\section{Field-contribution to $\xi_{\mathrm{c}}$ in BDFT}

Practically all Kohn-Sham BDFT calculations of magnetizabilities use standard exchangecorrelation functionals, ignoring the field dependence. Having studied the errors of typical DFAs, we now consider the importance of the field dependence of the correlation functional $E_{\mathrm{c}}(\rho, \mathbf{B})$ in BDFT. In Refs. 6 and 37 we studied in a similar manner the importance of the current contribution to the correlation functional $E_{\mathrm{c}}\left(\rho, \mathbf{j}_{\mathrm{p}}\right)$ in CDFT, for NMR constants.

In Table 4, we have, for a number of systems, calculated the $\operatorname{CCSD}(\mathrm{T})$ magnetizability $\xi_{\mathrm{CC}}$ and its correlation contribution, as obtained by subtracting the Hartree-Fock magnetizability $\xi_{\mathrm{HF}}$. The $\operatorname{CCSD}(\mathrm{T})$ correlation contribution of the magnetizability $\xi_{\mathrm{corr}}=\xi_{\mathrm{CC}}-\xi_{\mathrm{HF}}$ is to a good approximation equal to the correlation contribution $\xi_{\mathrm{c}}$ in BDFT. To estimate the field contribution to $\xi_{\mathrm{c}}$, we have calculated, by the Lieb variation principle, the KohnSham potential, orbitals, and orbital energies corresponding to the $\operatorname{CSDD}(\mathrm{T})$ density. From these Kohn-Sham quantities, we have calculated the magnetizability $\xi_{\mathrm{KS}(\mathrm{CC})}$ by standard linear-response theory, neglecting all field dependence. Finally, the field contribution to $\xi_{\mathrm{c}}$ is

estimated as $\xi_{\mathrm{c}}^{\mathrm{B}}=\xi_{\mathrm{CC}}-\xi_{\mathrm{KS}(\mathrm{CC})}$. For the total magnetizability, we used the CFOUR code; ${ }^{38}$ for the Lieb calculation, the DALTON code. ${ }^{39,40}$

For the noble gas atoms $\mathrm{He}$ and $\mathrm{Ne}, \xi_{\mathrm{c}}^{\mathrm{B}}=0$. For the molecules studied, $\xi_{\mathrm{c}}^{\mathrm{B}}$ ranges from about $7 \%$ of the total correlation contribution for the HF molecule to $66 \%$ for $\mathrm{CO}$ and $154 \%$ for $\mathrm{N}_{2}$. Clearly, the accurate calculation of magnetizabilities in Kohn-Sham theory is only possible with the inclusion of a field dependence in the correlation functional $E_{\mathrm{c}}(\rho, \mathbf{B})$.

These results are consistent with previous work ${ }^{5}$ and consistent with the magnitude of induced currents in these molecules - see, for example, Ref. 41 for plots of induced currents. 
Table 4: The CCSD(T)/aug-cc-pVQZ magnetizability $\xi_{\mathrm{CC}}$, the $\operatorname{CCSD}(\mathrm{T})$ correlation contribution $\xi_{\text {corr }}$, and the field contribution $\xi_{\mathrm{c}}^{\mathrm{B}}$ to the BDFT correlation term $\xi_{\mathrm{c}}$. In the first column, the total magnetizability is for comparison given in SI units $\left(\mathrm{JT}^{-2} 10^{-30}\right)$, the remaining numbers are in atomic units.

\begin{tabular}{l|c|ccc}
\hline \hline & $\xi_{\mathrm{CC}}(\mathrm{SI})$ & $\xi_{\mathrm{CC}}$ & $\xi_{\text {corr }}^{\mathrm{tot}}$ & $\xi_{\mathrm{c}}^{\mathrm{B}}$ \\
\hline $\mathrm{He}$ & -31.42 & -0.398 & -0.003 & 0.0 \\
$\mathrm{Ne}$ & -126.0 & -1.597 & -0.035 & 0.0 \\
$\mathrm{HF}$ & -176.9 & -2.242 & -0.055 & -0.004 \\
$\mathrm{H}_{2} \mathrm{O}$ & -236.1 & -2.992 & -0.062 & -0.010 \\
$\mathrm{NH}_{3}$ & -291.2 & -3.643 & -0.048 & -0.012 \\
$\mathrm{CH}_{4}$ & -319.7 & -4.052 & -0.077 & -0.045 \\
$\mathrm{CO}$ & -210.9 & -2.673 & -0.082 & -0.054 \\
$\mathrm{~N}_{2}$ & -206.2 & -2.613 & -0.045 & -0.068 \\
\hline \hline
\end{tabular}

Around the hydrogen atoms, the currents are weak such that the systems depend only weakly on the current corrections.

\section{Adiabatic connection in a magnetic field}

In this section, we consider the adiabatic connection for molecules in a magnetic field. We begin by calculating and modelling accurate FCI AC curves of $\mathrm{H}_{2}$ and $\mathrm{LiH}$ in Sec. (at the zero-field equilibrium geometry) and in Sec. (at the field optimized geometry). Finally, in Sec., the performance of some standard DFAs is analysed by comparing their AC curves with the corresponding FCI curves.

\section{FCI AC curves at a fixed geometry}

In Fig. 3, we have plotted $\mathrm{AC}$ curves for $\mathrm{H}_{2}$ at its zero-field equilibrium geometry, in a perpendicular magnetic field of different field strengths $B$. With increasing field strength, the curves become more bent, indicative of increasing importance of static correlation. The explanation of this behaviour is as follows: In the magnetic field, the atomic densities contract, decreasing the overlap between the two atoms and reducing the equilibrium bond length. ${ }^{4}$ The increased field thus effectively stretches the bond, increasing static correlation.

We now apply the AC model developed by Teale, Helgaker, and Coriani (THC) in Ref. 
Table 5: Field dependence of the THC parameters $s$ and $a$ for the global fit (Fit 1) and end-point fit where $s=\mathcal{W}_{\mathrm{THC}}^{\prime}(0)$ and $a=\mathcal{W}_{\mathrm{THC}}(1)$ (Fit 2).

\begin{tabular}{cc|ccccc}
\hline \hline & & 0.0 & 0.2 & 0.4 & 0.6 & 0.8 \\
\hline \multirow{2}{*}{ Fit 1 } & $a$ & -0.192 & -0.195 & -0.202 & -0.211 & -0.219 \\
& $s$ & -0.131 & -0.134 & -0.142 & -0.153 & -0.167 \\
\hline \multirow{2}{*}{ Fit 2 } & $a$ & -0.208 & -0.211 & -0.220 & -0.230 & -0.241 \\
& $s$ & -0.125 & -0.128 & -0.135 & -0.145 & -0.157 \\
\hline \hline
\end{tabular}

18. The THC AC integrand is given by

$$
\mathcal{W}_{\mathrm{THC}}(\lambda)=\frac{a s \lambda(4 a+s \lambda)}{(2 a+s \lambda)^{2}}
$$

where $s=\mathcal{W}_{\mathrm{THC}}^{\prime}(0)$ is the initial slope and $a=\mathcal{W}_{\mathrm{THC}}(\infty)$ is the asymptotic value in the strongly interacting limit. The parameters $a$ and $s$ may be fitted for $\mathcal{W}_{\mathrm{THC}}(\lambda)$ to reproduce $\mathcal{W}(\lambda)$ calculated $a b$ initio. The THC model performs best when $a$ and $s$ are fitted globally, but a good agreement is also obtained by adjusting $s$ and $a$ to give $\mathcal{W}_{\mathrm{THC}}^{\prime}(0)=\mathcal{W}^{\prime}(0)$ and $\mathcal{W}_{\mathrm{THC}}(1)=\mathcal{W}(1)$, which is achieved by setting $s=\mathcal{W}^{\prime}(0)$ and then

$$
a=\frac{s^{2}-4 s t+s \sqrt{s^{2}+8 s t}}{8(t-s)}
$$

with $t=\mathcal{W}(1)$. Although based on Görling-Levy perturbation theory, the THC model is able to capture static correlation. ${ }^{18}$

In Fig. 3, the globally fitted AC curve is hardly visible due to its good agreement with the exact AC curve, whereas the curve with $s=\mathcal{W}^{\prime}(0)$ and $a=\mathcal{W}(1)$ deviates slightly but is still very close, capturing the correct overall behaviour-namely, the increase of static correlation with increasing field strength. The end-point fitted curve becomes only marginally worse with increasing magnetic field strength. For longer bond lengths of 3 and $5 \mathrm{bohr}$, the model performs slightly worse, but this is already true at zero field strength (see Ref. 18) and not primarily an effect of the magnetic field. 
Table 5 shows how the THC parameters change with increasing magnetic field. The $s$ parameter agrees better between the two fits than does the $a$ parameter. The second (physically more justified) fit reveals how the initial AC slope $s$ becomes more negative with increasing magnetic field. The slope increases in magnitude more strongly in stronger magnetic fields.

\section{FCI AC curves at optimized geometries}

At a fixed geometry, the effective bond stretching leads to an increased curvature of the AC curves with increasing field strength, corresponding to an increase of static correlation. This effect is removed by calculating the AC curve at the optimized equilibrium geometry for each field strength. Indeed, Fig. 4 reveals that, when studied at the field-dependent equilibrium geometry, the AC curve is not much affected by the magnetic field. Hence, the magnetic field does not affect electron correlation appreciably - in particular, no static correlation is introduced.

Integrating the $\mathrm{AC}$ curves $\mathcal{W}(\lambda)$ from $\lambda=0$ to $\lambda=1$, we obtain the correlation energy. From zero field to a field strength of $0.6 B_{0}$, the correlation energy of $\mathrm{H}_{2}$ changes by only $-0.002 E_{\mathrm{h}}$. The corresponding change in the kinetic correlation energy is about $0.002 E_{\mathrm{h}}$, as expected in the dynamical correlation regime, in which $T_{\mathrm{c}}(\rho) \approx-E_{\mathrm{c}}(\rho)$.

\section{DFA AC curves at optimized geometry}

In this section, we consider $\mathrm{AC}$ curves for molecules in a strong magnetic field for some standard DFAs. In Fig. 5, AC curves for $\mathrm{H}_{2}$ and $\mathrm{LiH}$ at $B=0$ and $B=0.6 B_{0}$ for several DFAs are compared with the corresponding FCI curve for $\mathrm{H}_{2}$ and the coupled-cluster doubles (CCD) curve for LiH. The DFA curves have been calculated using the self-consistently optimized DFA ground-state density. Calculations on $\mathrm{H}_{2}$ showed that use of the FCI density (in place of the self-consistent DFA density) has no visible impact on the $\mathrm{AC}$ curves.

The plots show that the behaviour of the AC curves is similar for the two field strengths, 
the main difference being that the initial slope of the curve is slightly larger in the higher field. Among the DFAs, the TPSS functional performs best relative to FCI theory, while the LYP curve shows a considerably poorer curvature than the other DFAs.

For $\mathrm{LiH}$, all DFAs have a too large initial slope. For $\mathrm{H}_{2}$, the PBE and $\operatorname{TPSS}\left(\tau_{\text {phys }}\right)$ initial slopes are slightly more negative than the FCI slope, while the LYP slope is more positive, both with and without magnetic field. At $0.8 B_{0}$, the FCI slope becomes slightly more negative. Since the AC curve integrates to the total correlation energy, a more negative initial slope is necessary for an increased absolute correlation energy. This behaviour is correctly captured by the PBE and $\operatorname{TPSS}\left(\tau_{\text {phys }}\right)$ functionals but not by the LYP functional.

To summarize, our calculations show that the errors present already at zero field outweigh those introduced by the field, suggesting that, for the performance in magnetic fields, the benefits obtained by improving the field-free case would be greater than improvements focusing on the effect of the field itself.

\section{Conclusions}

The extensions of DFT to systems in the presence of magnetic fields, magnetic-field densityfunctional theory (BDFT) and current density-functional theory (CDFT), were introduced within the framework of convex analysis. In particular, the four-way correspondence of saddle functions and their concave and convex parents was used to elucidate the relationships between these alternative DFT approaches to molecules in a magnetic field. The KohnSham decompositions of the density functionals in BDFT and CDFT were compared and a relationship between their exchange--correlation functionals was established; the BDFT exchange--correlation energy being an upper bound to the CDFT exchange-correlation energy.

The effect of a magnetic field on the Kohn-Sham energy components in BDFT was studied using high-level ab initio theory. In the weak-field regime, the second derivative of the energy with respect to the magnetic field (i.e., the negative magnetizability) and its contributions 
were studied in detail. Our calculations highlighted the fact that present DFAs give poor charge densities, suggesting that significant improvements can only be obtained by developing DFAs with improved self-consistent densities. For the molecules studied, the field-dependence of the BDFT correlation energy contributes up to $2.6 \%$ of the total magnetizability (for $\mathrm{N}_{2}$ ), which is comparable to the total correlation contribution. Hence, once accurate charge densities are achieved, field-dependent contributions cannot be neglected for high-accuracy calculations.

To analyse the role of electron correlation in BDFT further, we studied the AC integrand at different field strengths. At a fixed molecular geometry, a compression of the charge density with increasing field strength leads to an increased amount of static correlation (increased curvature of the AC curve). However, this effect is relatively subtle and existing models for the $\mathrm{AC}$ integrand are able to capture this behaviour. For the DFAs considered here, the most accurate $\mathrm{AC}$ integrands were provided by generalized meta-GGA functionals in the context of BDFT, consistent with previous findings for CDFT. ${ }^{7}$ However, the dominant errors in the correlation functionals are those already present in the absence of a field, indicating the need for an improvement in the parent zero-field functionals and their associated charge densities before the benefit of field-dependent corrections can be realized.

\section{Acknowledgements}

This work was supported by the Norwegian Research Council through the CoE Centre for Theoretical and Computational Chemistry (CTCC) Grant Nos. 179568/V30 and 171185/V30 and through the European Research Council under the European Union Seventh Framework Program through the Advanced Grant ABACUS, ERC Grant Agreement No. 267683. AMT is grateful for support from a Royal Society University Research Fellowship and the Engineering and Physical Sciences Research Council EPSRC, Grant No. EP/M029131/1. 


\section{References}

(1) Wickramasinghe, D. T.; Ferrario, L. Magnetism in Isolated and Binary White Dwarfs. PASP 2000, 112, 873.

(2) Price, D. J.; Rosswog, S. Producing Ultrastrong Magnetic Fields in Neutron Star Mergers. Science 2006, 312, 719-722.

(3) Gourgouliatos, K. N.; Wood, T. S.; Hollerbach, R. Magnetic field evolution in magnetar crusts through three-dimensional simulations. Proc. Nat. Acad. Sci. 2016, 113, $3944-$ 3949.

(4) Lange, K. K.; Tellgren, E. I.; Hoffmann, M. R.; Helgaker, T. A Paramagnetic Bonding Mechanism for Diatomics in Strong Magnetic Fields. Science 2012, 337, 327-331.

(5) Lutnæs, O. B.; Teale, A. M.; Helgaker, T.; Tozer, D. J.; Ruud, K.; Gauss, J. Benchmarking density-functional-theory calculations of rotational $g$ tensors and magnetizabilities using accurate coupled-cluster calculations. J. Chem. Phys. 2009, 131, 144104.

(6) Teale, A. M.; Lutnæs, O. B.; Helgaker, T.; Tozer, D. J.; Gauss, J. Benchmarking density-functional theory calculations of NMR shielding constants and spin-rotation constants using accurate coupled-cluster calculations. J. Chem. Phys. 2013, 138.

(7) Furness, J. W.; Verbeke, J.; Tellgren, E. I.; Stopkowicz, S.; Ekström, U.; Helgaker, T.; Teale, A. M. Current Density Functional Theory Using Meta-Generalized Gradient Exchange-Correlation Functionals. J. Chem. Theory Comput. 2015, 11, 4169-4181.

(8) Vignale, G.; Rasolt, M. Density-functional theory in strong magnetic fields. Phys. Rev. Lett. 1987, 59, 2360-2363.

(9) Vignale, G.; Rasolt, M. Current- and spin-density-functional theory for inhomogeneous electronic systems in strong magnetic fields. Phys. Rev. B 1988, 37, 10685-10696. 
(10) Grayce, C. J.; Harris, R. A. Magnetic-field density-functional theory. Phys. Rev. A 1994, 50, 3089-3095.

(11) Becke, A. D. A new mixing of Hartree-Fock and local densityfunctional theories. J. Chem. Phys. 1993, 98, 1372-1377.

(12) Ernzerhof, M. Construction of the adiabatic connection. Chem. Phys. Lett. 1996, 263, $499-506$.

(13) Seidl, M.; Perdew, J. P.; Kurth, S. Simulation of All-Order Density-Functional Perturbation Theory, Using the Second Order and the Strong-Correlation Limit. Phys. Rev. Lett. 2000, 84, 5070 .

(14) Peach, M. J. G.; Teale, A. M.; Tozer, D. J. Modeling the adiabatic connection in H2. J. Chem. Phys. 2007, 126.

(15) Peach, M. J. G.; Miller, A. M.; Teale, A. M.; Tozer, D. J. Adiabatic connection forms in density functional theory: $\mathrm{H} 2$ and the He isoelectronic series. J. Chem. Phys. 2008, 129.

(16) Gori-Giorgi, P.; Vignale, G.; Seidl, M. Electronic Zero-Point Oscillations in the StrongInteraction Limit of Density Functional Theory. J. Chem. Theory Comput. 2009, 5, 743.

(17) Kim, J.; Jung, Y. Analytical Double-Hybrid Density Functional Based on the Polynomial Series Expansion of Adiabatic Connection: A Quadratic Approximation. J. Chem. Theory Comput. 2015, 11, 45-54.

(18) Teale, A. M.; Coriani, S.; Helgaker, T. Accurate calculation and modeling of the adiabatic connection in density functional theory. J. Chem. Phys. 2010, 132, 164115.

(19) Lieb, E. H. Density functionals for coulomb systems. Int. J. Quantum Chem. 1983, 24, 243. 
(20) Lammert, P. E. Differentiability of Lieb functional in electronic density functional theory. Int. J. Quantum Chem. 2007, 10\%, 1943.

(21) Levy, M. Universal Variational Functionals od Electron-Densities, 1st-order DensityMatrices, and Natural Spin-Orbitals and Solutions of the V-representability Problem. Proc. Natl. Acad. Sci. U.S.A. 1979, 76, 6062.

(22) van Tiel, J. Convex Analysis: An Introductory Text; Wiley, 1984.

(23) Barbu, V.; Precupano, T. Convexity and Optimization in Banach Spaces, 4th ed.; Springer, 2014.

(24) Tellgren, E. I.; Kvaal, S.; Sagvolden, E.; Ekström, U.; Teale, A. M.; Helgaker, T. Choice of basic variables in current-density-functional theory. Phys. Rev. A 2012, 86, 062506.

(25) Lieb, E. H.; Schrader, R. Current densities in density-functional theory. Phys. Rev. A 2013, 88, 032516.

(26) Rockafellar, R. T. A general correspondence between dual minimax problems and convex programs. Pacific J. Math. 1968, 25, 597-611.

(27) Levy, M.; Perdew, J. P. Hellmann-Feynman, virial, and scaling requisites for the exact universal density functionals. Shape of the correlation potential and diamagnetic susceptibility for atoms. Phys. Rev. A 1985, 32, 2010-2021.

(28) Maximoff, S. N.; Scuseria, G. E. Nuclear magnetic resonance shielding tensors calculated with kinetic energy density-dependent exchange-correlation functionals. Chem. Phys. Lett. 2004, 390, 408-412.

(29) Tellgren, E. I.; Soncini, A.; Helgaker, T. Nonperturbative ab initio calculations in strong magnetic fields using London orbitals. J. Chem. Phys. 2008, 129, 154114.

(30) LONDON, an ab-initio program package for calculations in finite magnetic fields, founded by E. I. Tellgren, A. Soncini and T. Helgaker. Programming by E. I. Tellgren (main 
programmer), K. K. Lange, A. M. Teale, U. E. Ekström, S. Stopkowicz and J. H. Austad. 2016.

(31) Ekström, U.; Visscher, L.; Bast, R.; Thorvaldsen, A. J.; Ruud, K. Arbitrary-Order Density Functional Response Theory from Automatic Differentiation. J. Chem. Theory Comput. 2010, 6, 1971-1980.

(32) London, F. Théorie quantique des courants interatomiques dans les combinaisons aromatiques. J. Phys. Radium 1937, 8, 397-409.

(33) Dunning, T. H. Gaussian basis sets for use in correlated molecular calculations. I. The atoms boron through neon and hydrogen. J. Chem. Phys. 1989, 90, 1007-1023.

(34) Woon, D. E.; Dunning, T. H. Gaussian basis sets for use in correlated molecular calculations. IV. Calculation of static electrical response properties. J. Chem. Phys. 1994, 100, 2975-2988.

(35) Wu, Q.; Yang, W. A direct optimization method for calculating density functionals and exchange-correlation potentials from electron densities. J. Chem. Phys. 2003, 118, $2498-2509$.

(36) Teale, A. M.; Coriani, S.; Helgaker, T. The calculation of adiabatic-connection curves from full conguration-interaction densities: Two-electron systems. J. Chem. Phys. 2009, $130,104111$.

(37) Reimann, S.; Ekström, U.; Stopkowicz, S.; Teale, A. M.; Borgoo, A.; Helgaker, T. The importance of current contributions to shielding constants in density-functional theory. Phys. Chem. Chem. Phys. 2015, 17, 18834-18842.

(38) CFOUR, a quantum chemical program package written by J.F. Stanton, J. Gauss, M.E. Harding, P.G. Szalay with contributions from A.A. Auer, R.J. Bartlett, U. Benedikt, C. Berger, D.E. Bernholdt, Y.J. Bomble, L. Cheng, O. Christiansen, M. Heckert, O. 
Heun, C. Huber, T.-C. Jagau, D. Jonsson, J. Jusélius, K. Klein, W.J. Lauderdale, D.A. Matthews, T. Metzroth, L.A. Mück, D.P. O’Neill, D.R. Price, E. Prochnow, C. Puzzarini, K. Ruud, F. Schiffmann, W. Schwalbach, C. Simmons, S. Stopkowicz, A. Tajti, J. Vázquez, F. Wang, J.D. Watts and the integral packages MOLECULE (J. Almlöf and P.R. Taylor), PROPS (P.R. Taylor), ABACUS (T. Helgaker, H.J. Aa. Jensen, P. Jørgensen, and J. Olsen), and ECP routines by A. V. Mitin and C. van Wüllen. For the current version, see http://www.cfour.de. 2016.

(39) Dalton, a molecular electronic structure program, Release Dalton2015.0 (2015), see http://daltonprogram.org. 2016.

(40) Aidas, K. et al. The Dalton quantum chemistry program system. WIREs Comput. Mol. Sci. 2015, 4, 269-284.

(41) Soncini, A.; Teale, A. M.; Helgaker, T.; Proft, F. D.; Tozer, D. J. Maps of current-density using density-functional methods. J. Chem. Phys. 2008, 129, 074101. 


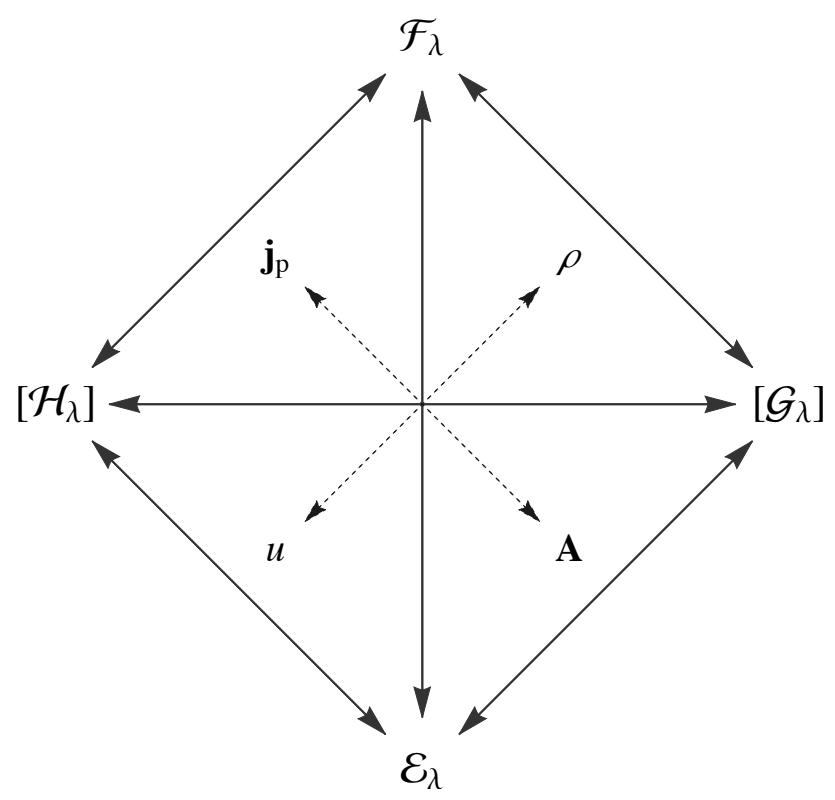

Figure 1: The four-way correspondence of DFT in a magnetic field, relating the concave function $\mathcal{E}_{\lambda}$, the convex function $\mathcal{F}_{\lambda}$, the equivalence class of concave-convex saddle functions $\left[\mathcal{G}_{\lambda}\right]$, and the equivalence class of convex-concave saddle functions $\left[\mathcal{H}_{\lambda}\right]$. Each function depends on the two adjacent variables. The down conjugations $\rho \rightarrow u$ and $\mathbf{j}_{\mathrm{p}} \rightarrow \mathbf{A}$ are minimizations with positive pairings $+(u \mid \rho)$ and $+\left(\mathbf{A} \mid \mathbf{j}_{\mathrm{p}}\right)$, respectively, while the up conjugations $u \rightarrow \rho$ and $\mathbf{A} \rightarrow \mathbf{j}_{\mathrm{p}}$ are maximizations with negative pairings $-(u \mid \rho)$ and $-\left(\mathbf{A} \mid \mathbf{j}_{\mathrm{p}}\right)$, respectively. The Hohenberg-Kohn variation principle corresponds in CDFT to the full conjugation $\left(\rho, \mathbf{j}_{\mathrm{p}}\right) \rightarrow(u, \mathbf{A})$ from $\mathcal{F}_{\lambda}$ to $\mathcal{E}_{\lambda}$ and in BDFT to the partial conjugation $\rho \rightarrow u$ from $\mathcal{G}_{\lambda}$ to $\mathcal{E}_{\lambda}$. 


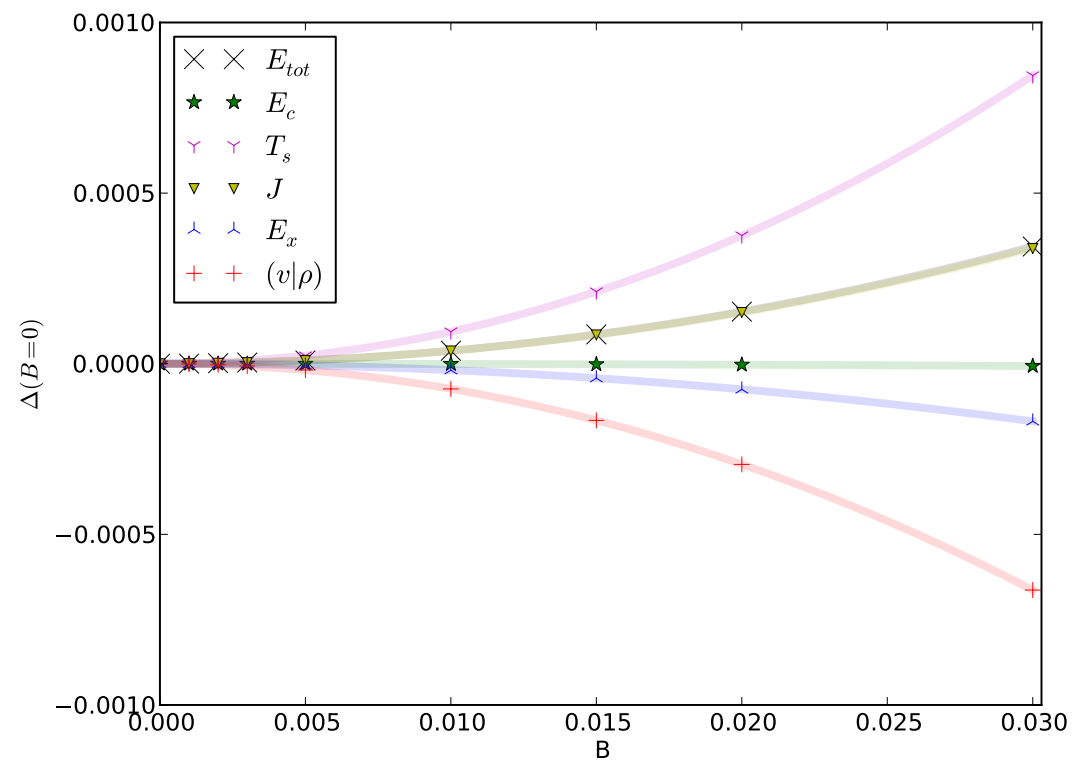

Figure 2: The Kohn-Sham energy components of $\mathrm{H}_{2}$ relative to the zero-field values as a function of the magnetic field strength (atomic units). The curves show the second-order approximation to the energy components (see text for details), with curvatures equal to the negative magnetizability at zero field (and its Kohn-Sham components). All calculations have been carried out at the FCI/aug-cc-pVTZ level of theory, at the zero-field equilibrium geometry. 


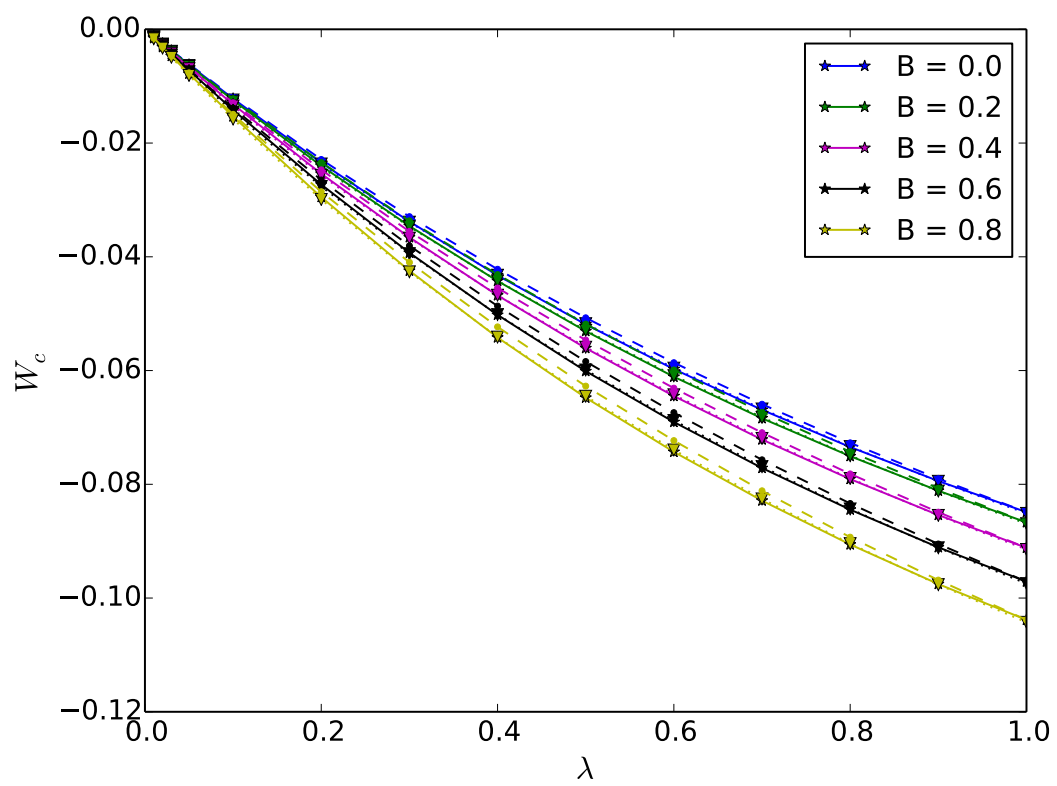

Figure 3: $\mathrm{AC}$ curves for $\mathrm{H}_{2}$ at different magnetic field strengths with fixed bond length 2.1 bohr (1.5 times the zero-field equilibrium bond length). The full lines show accurate ab initio AC integrands using the FCI density for each magnetic field strength. The dashed lines correspond to the THC model in Eq. (58) using $s=\mathcal{W}^{\prime}(0)$ and $a=\mathcal{W}(1)$. The dotted lines show the same model with globally fitted parameters $s$ and $a$, which are nearly on top of the accurate curves.
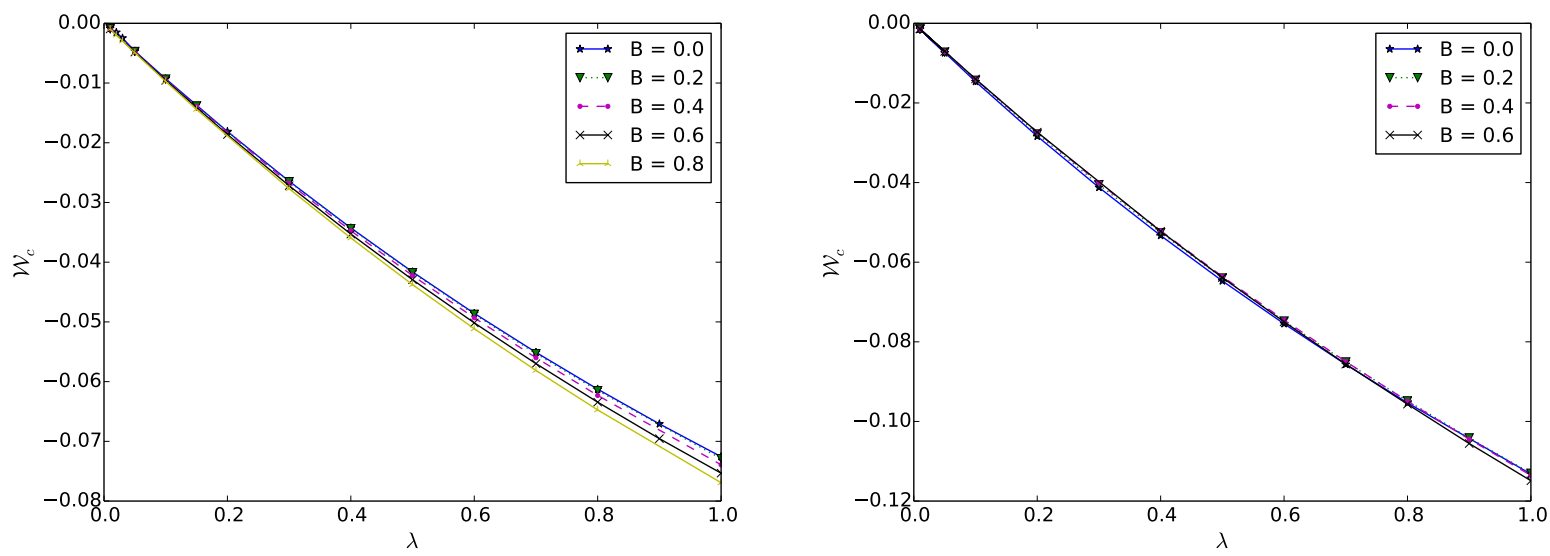

(a) $\mathrm{H}_{2}$, all calculations at the FCI / aug-cc-pVTZ (b) LiH, all calculations at the CCD / aug-cc-pVTZ level. level.

Figure 4: $\mathrm{AC}$ curves of $\mathrm{H}_{2}$ and $\mathrm{LiH}$ in a perpendicular magnetic field (atomic units). Each curve is calculated at the equilibrium bond distance in the applied field. 


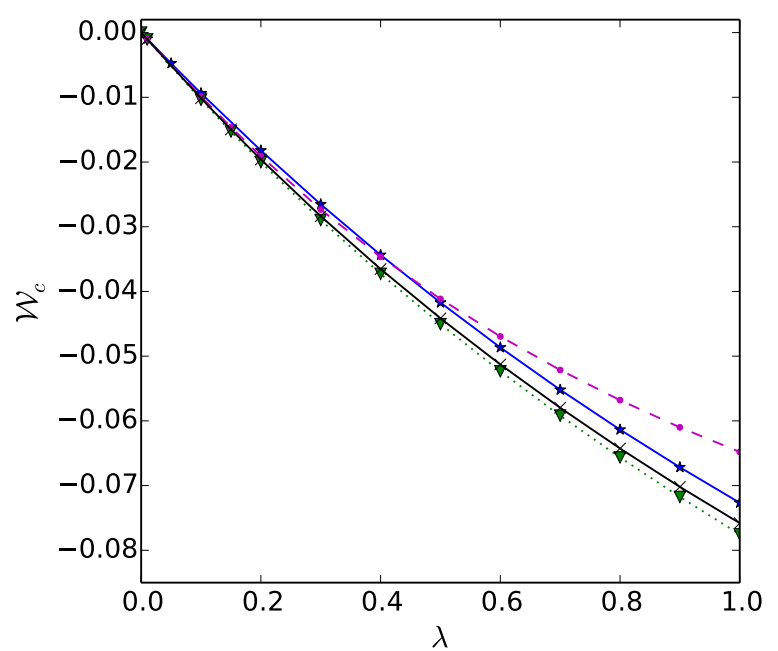

(a) $\mathrm{H}_{2}, \mathrm{~B}=0.0$ (a.u.)

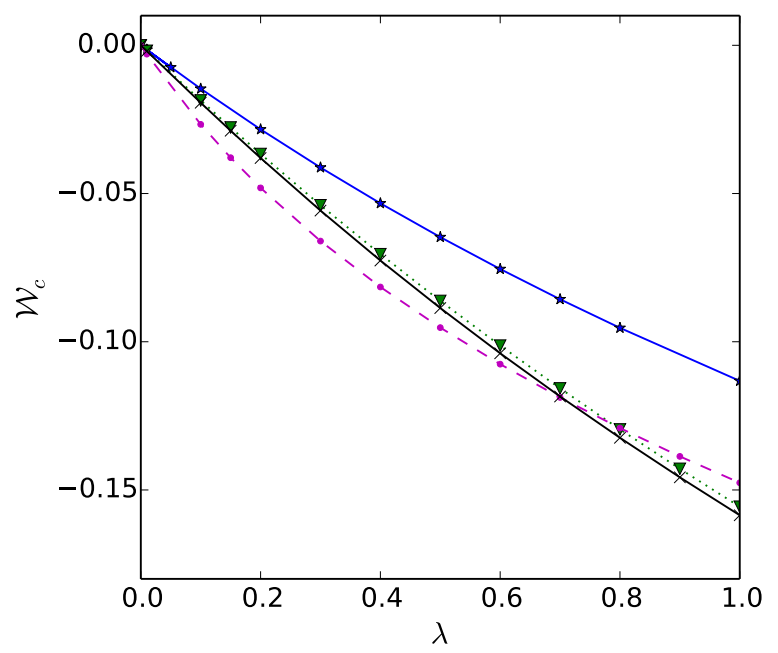

(c) $\mathrm{LiH}, \mathrm{B}=0.0$ (a.u.)

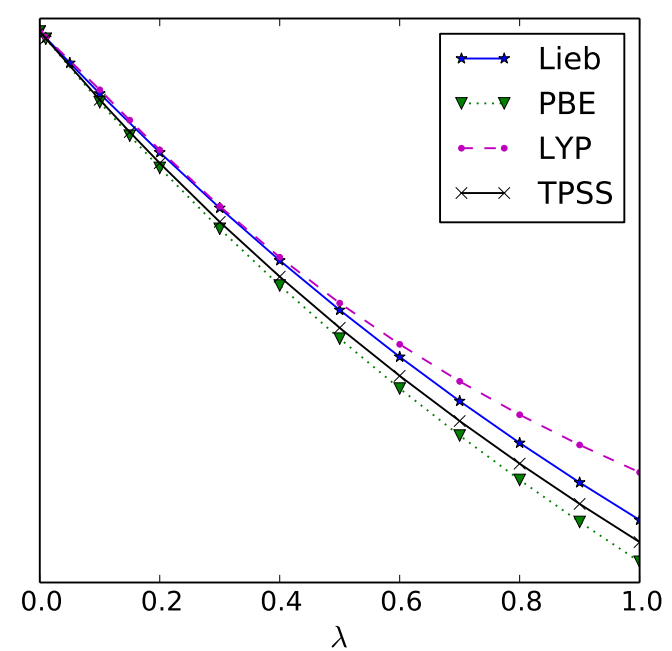

(b) $\mathrm{H}_{2}, \mathrm{~B}=0.6$ (a.u.)

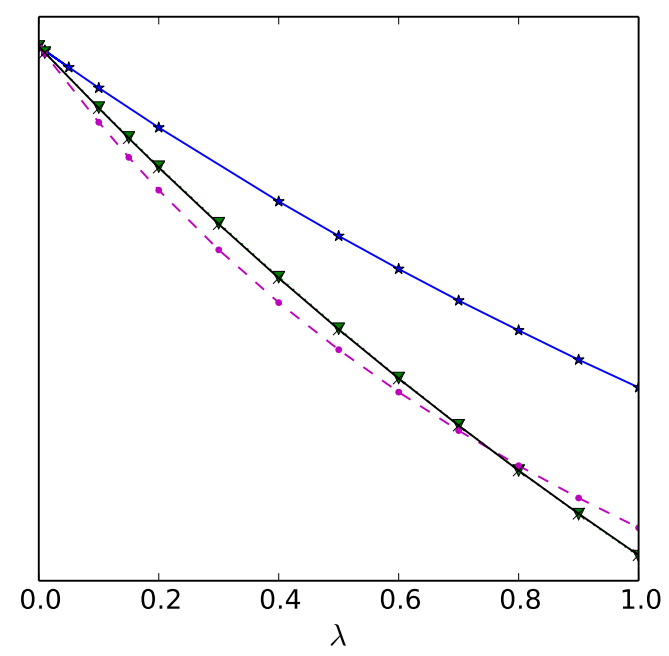

(d) $\mathrm{LiH}, \mathrm{B}=0.6$ (a.u.)

Figure 5: $\mathrm{AC}$ curves for $\mathrm{H}_{2}$ and $\mathrm{LiH}$ with optimized density at each field strength (atomic units). For the DFA calculations the self-consistent density is used. For the ab initio calculations for $\mathrm{H}_{2}$ the FCI density is used and for $\mathrm{LiH}$ the CCD density is used. 